\title{
Fluorofenidone attenuates pulmonary inflammation and fibrosis via inhibiting the IL-11/MEK/ERK signaling pathway
}

\section{Cheng Song ( $\nabla$ songchengedu@sina.com )}

Central Hospital of Wuhan, Huazhong University of Science and Technology

Xiaofan Liu

Central Hospital of Wuhan, Huazhong University of Science and Technology

\section{Weijun Tan}

Central Hospital of Wuhan, Huazhong University of Science and Technology

\section{Xiaoqun Guo}

Central Hospital of Wuhan, Huazhong University of Science and Technology

\section{Yahui Mao}

Central Hospital of Wuhan, Jiang Han University

\section{Qi Zhou}

Central Hospital of Wuhan, Jiang Han University

\section{Shuang Geng}

Central Hospital of Wuhan, Huazhong University of Science and Technology Yi Hu

Central Hospital of Wuhan, Huazhong University of Science and Technology

\section{Research Article}

Keywords: Fluorofenidone, Pulmonary inflammation, Pulmonary fibrosis, Interleukin-11, IL-11/MEK/ERK signaling pathway

Posted Date: February 16th, 2022

DOI: https://doi.org/10.21203/rs.3.rs-1358661/v1

License: (c) (i) This work is licensed under a Creative Commons Attribution 4.0 International License. Read Full License 


\section{Abstract}

Background: Idiopathic pulmonary fibrosis (IPF) is a special type of interstitial lung diseases with unknown cause. Interleukin (IL)-11 is a new therapeutically target for fibrosis diseases. In this experiment, we further explored the pharmacology mechanism and molecular target of fluorofenidone (FD) in vivo and in vitro. Methods: Male C57BL/6J mice were intratracheally injected with Bleomycin (BLM) or saline. FD was administered throughout the course of the experiment. Lung tissue sections were stained with haemotoxylin and eosin, Masson's trichrome and Immunohistochemistry. Cytokines were measured by ELISA, and phosphorylated extracellular signal-regulated kinase (p-ERK), phosphorylated mitogenactivated protein kinase (p-MEK), IL-11RA and gp130 were measured by Western blot. The RAW264.7 cells and the normal human lung fibroblasts (NHLFs) were treated with IL-11 and/or FD, IL-11RA-siRNA, MEK Inhibitor. The expressions of p-ERK, p-MEK, IL-11RA, gp130, a-SMA, fibronectin and collagen $\rrbracket$ were measured by Western blot and/or RT-PCR, the cytokines were measured by ELISA. Results: FD attenuated pulmonary inflammation and fibrosis, and decreased the expressions of IL-8, IL-18, IL-11, MCP-1, p-ERK and p-MEK in the bleomycin mouse model. Moreover, the protein expressions of IL-11RA and gp130 were inhibited by FD in IL-11-induced RAW264.7 cells. Additionally, FD attenuated IL-11-induced expressions of p-ERK and p-MEK in IL-11-induced RAW264.7 cells and IL-11-induced normal human lung fibroblasts. Meanwhile, FD reduced IL-11-induced the levels of IL-8, IL-18 and MCP-1, blocked IL-11-induced the activation of fibroblasts. Conclusion: These findings demonstrated that FD attenuates BLM-induced pulmonary inflammation and fibrosis in mice via inhibiting the IL-11/MEK/ERK signaling pathway.

\section{Introduction}

Idiopathic pulmonary fibrosis (IPF) is a progressive and devastating lung disease of with the worst prognosis-the mortality rate at 3 to 5 years after diagnosis is $50 \%$, 2 . The misdiagnosis rate of IPF and the increasing rate of deaths due to IPF aggravate the global medical burden. In the tissue injury phase, the pro-inflammatory cytokines that are secreted from macrophages, tissue fibroblasts, and other local cell populations have been identified as triggers of fibrosis 3,4 .

Interleukin (IL)-11, a pleiotropic cytokine, is thought to play a central role in fibrosis diseases, such as cardiovascular fibrosis, kidney fibrosis and pulmonary fibrosis ${ }^{5,6}$. The transition from fibroblast to myofibroblast in the heart and kidney was found to be dependent on an autocrine IL-11 signaling loop ${ }^{5}$. IL-11 is a member of the IL-6 family of cytokines. IL-11 can bind to free IL11RA, which is shed from CFs membranes and signal in trans in cells that express gp1305,7. The gp130 receptor subsequently triggers a number of different pathways, including canonical JAK/STAT signaling, as well as ERK and AKT ${ }^{8,9}$. Stuart A Cook and colleagues show that inhibition of IL-11 prevented fibroblast activation in the heart and kidneys in response to a range of profibrotic stimuli ${ }^{5}$. The expression of IL-11 is up-regulated in the fibroblasts and lung from IPF patients ${ }^{5,6}$. Intraperitoneal injection of neutralizing anti-IL-11 antibodies could alleviate pulmonary fibrosis in the BLM mouse model ${ }^{6}$. Additionally, the activation of ERK signaling in the lung is dependent on IL-11 signaling . 
Fluorofenidone [1-(3-fluorophenyl)-5-methyl-2-(1H)-pyridone] (FD), a new antifibrotic drug, was developed and patented by the Pharmaceutical School of Central South University ${ }^{10}$. Although our previous studies showed that administration of FD can attenuate pulmonary inflammation and fibrosis through different signaling pathways ${ }^{11,12,13}$, but the pharmacology mechanism and molecular target of FD are not well understood.

Hence, we further explore the antifibrotic mechanism of FD, and we found that FD could ameliorate pulmonary inflammation and fibrosis through inhibiting the IL-11/MEK/ERK signaling pathway in vivo and in vitro.

\section{Materials And Methods}

\section{Experimental Animals and Preparation}

The male C57BL/6J mice were purchased from Hubei Provincial Laboratory Animal Public Service Center. And the animal experiments were reviewed and approved by Laboratory Animal Center, Huazhong University of Science and Technology. Forty mice were randomly divided into five groups with each group includes eight mice: the control group (Control), the BLM group (BLM), the BLM/fluorofenidone group (BLM+FD), the BLM/Mouse IL-11 antibody group (BLM+anti-IL-11) and the BLM/MEK Inhibitor group (BLM+MEK Inhibitor). The model of pulmonary fibrosis was established as our previous studies ${ }^{13}$. Administration FD (500 mg/kg, once per day), intraperitoneal injection of mouse IL-11 antibody (100mg/kg, once per day, R\&D, USA), and intraperitoneal injection of MEK Inhibitor (8mg/kg, once per day, CST, USA) were executed $24 \mathrm{hrs}$ before establishment of the model. All the mice were killed at day 14 . The blood were collected using the method of removing eyeball. The lung tissues were collected and preserved for the following experiments.

\section{Cell Culture}

The RAW264.7 cells and the normal human lung fibroblasts (NHLFs) were grown in DMEM medium supplemented with $10 \%$ fetal bovine serum and $1 \%$ penicillin-streptomycin at $37^{\circ} \mathrm{C}$ in $5 \% \mathrm{CO} 2$. The RAW264.7 cells were pre-incubated with FD ( $2 \mathrm{mM})$ for $24 \mathrm{hrs}$. Subsequently, IL-11 (25ng/ml, R\&D, USA) and/or MEK Inhibitor (10uM, CST, USA) were added to the culture media for $6 \mathrm{hrs}$. The NHLFs were preincubated with FD (2mM) for 24 hrs. Subsequently, IL-11 (25ng/ml, Life Technologies, USA) and/or MEK inhibitor (10uM, CST, USA) was added to the culture media for $45 \mathrm{~min}$ or $48 \mathrm{hrs}$.

\section{Haematoxylin and Eosin Staining, Masson's Trichrome Staining and Immunohistochemistry}

The left lung tissues were fixed in $4 \%$ paraformaldehyde, and then embedded in paraffin. Tissue section (4um) was stained with hemotoxylin and eosin and Masson's Trichrome according to the manufacturers' protocols. The inflammatory score and fibrosis score were assigned using the criteria as Szapiel, S. V. et al. and Ashcroft, T. et al. described ${ }^{14,15}$. 
Immunohistochemistry was performed, as previously described ${ }^{16}$. Primary antibodies used included:


obtained at Central Hospital of Wuhan on a Nikon microscope, and quantified using Image-Pro Plus 6.0.

\section{Mediators Measurements by ELISA}

The concentrations of IL-8, IL-11, IL-18, and MCP-1 in blood of mice and in the supernatant fluid of the RAW264.7 cells were determined by ELISA, according to manufacturer's instructions (IL-8, Meimian, China; IL-11, Cusabio, China; MCP-1, Cusabio, China; IL-18, Cusabio, China).

\section{RNA Extraction and Real-time PCR Quantification}

Total RNA was isolated from NHLFs by Trizol Reagent according to the manufacturer's instructions (Invitrogen, Grand Island, NY). The mRNA levels of fibronectin, collagen \and a-SMA were measured by real-time PCR. The specific primers were the same as previous studies ${ }^{12}$.

\section{Western Blotting Analysis}

The proteins from Lung tissues or cells were extracted using RIPA buffer containing protease inhibitors, and then were boiled at $100^{\circ} \mathrm{C}$ for $10 \mathrm{~min}$. Protein concentrations were determined using the BCA Protein Assay Kit. For Western blot analysis, 20-50 $\mathrm{gg}$ of protein was separated on 8-12\% SDS-polyacrylamide gel under reducing conditions and transferred onto polyvinylidene difluoride membranes. The membranes were blocked and then incubated overnight at $4^{\circ} \mathrm{C}$ with primary antibodies against $\mathrm{p}$-ERK (CST, USA), ERK (CST, USA), p-MEK (Santa, USA), MEK (Santa, USA), IL-11RA (Bioswamp, China), gp130

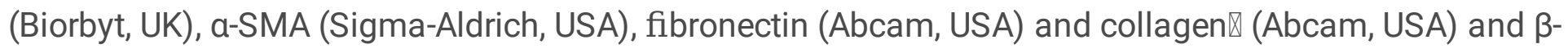
actin (Sigma-Aldrich, USA). The membranes were subsequently incubated with secondary antibodies, such as a goat anti-rabbit or goat anti-mouse horseradish peroxidase-conjugated antibody.

\section{siRNA Transfection}

The IL-11RA and negative-control siRNA were purchased (Ribobio, Co, Ltd, Guangzhou, China), and transfections of the RAW264.7 cells and the NHLFs were performed using the Lipofectamine Transfection Reagents. The culture medium was changed after $6 \mathrm{hrs}$, and then the cells were maintained at $37^{\circ} \mathrm{C}$ in $5 \%$ CO2 for $18 \mathrm{hrs}$. Subsequently, the cells could be used in the following experiments.

\section{Statistical Analysis}

All data were expressed as the mean \pm standard error. One-way analysis of variance and post hoc multiple-comparison tests was used for analyzing statistical differences among different groups. $\mathrm{P} \bowtie 0.05$ was considered significant.

\section{Results}


Our previous studies showed that BLM could induce severe alveolitis and fibrosis in mouse model. The principal phenotype of alveolitis were the aggregation of a large number of inflammatory cells, mainly including neutrophils and mononuclear cells; and the destruction and repair with thickening of alveolar septa (Fig. 1B). Meanwhile, the pathological manifestations of severe fibrosis were distorted pulmonary architecture and excessive deposition of collagen in the interstitium (Fig. 1H). As in previous research, we found that FD ameliorated pulmonary inflammation and fibrosis in this experiment (Fig. 1C, F, I, L). Besides, intraperitoneal injection of neutralizing anti-IL-11 antibodies or MEK inhibitor also reduced lung inflammation and fibrosis (Fig. 1D-F, J-L).

\section{Fluorofenidone Ameliorated the Expressions of Fibronectin, Collagen $\triangle$ and $a-S M A$ in the Bleomycin Mouse Model}

Microscopic investigations of immunohistochemical staining showed that the protein expressions of fibronectin, collagen $\otimes$ and a-SMA increased significantly in the bleomycin mouse model, which were remarkably attenuated by FD, neutralizing anti-IL-11 antibodies or MEK inhibitor (Fig. 2A-D). No significant difference was observed among FD, neutralizing anti-IL-11 antibodies and MEK inhibitor groups.

\section{Fluorofenidone Decreased the Levels of Inflammatory Factors and Chemokine Both in vivo and in vitro}

Inflammation persists in the whole process of pulmonary fibrosis. We found that the expressions of IL-8 (Fig. 3A), IL-18 (Fig. 3B), MCP-1 (Fig. 3C) and IL-11 (Fig. 3D) were significantly increased in the bleomycin mouse model than control mouse model, which were notably suppressed by FD, neutralizing anti-IL-11 antibodies or MEK inhibitor.

Moreover, ELISA analysis showed that the levels of IL-8, IL-18 and MCP-1 were markedly higher in the supernatant fluid of RAW264.7 cells treated with IL-11 compared with the controls, and FD pre-treatment, IL-11RA-siRNA transfection or MEK inhibitor treatment significantly prevented the increase in the expressions of IL-8, IL-18 and MCP-1 (Fig. 7A-C).

\section{Fluorofenidone Down-regulated the Expressions of IL-11RA and gp130 Only in RAW264.7 cells}

Current studies have found that IL- 11 plays a more important role than TGF - $\beta 1$ in the process of fibrosis, and we found that FD significantly attenuated BLM-induced the protein expressions of IL-11 in mice lung tissues. Then we explored whether FD could inhibit levels of IL-11RA and gp130 in vivo and in vitro.

The protein expressions of IL-11RA and gp130 after treatment with BLM were higher in the lungs compared with control group, but were not influenced by FD, neutralizing anti-IL-11 antibodies or MEK inhibitor (Fig. 4D-F). And we found that the levels of IL-11RA and gp130 were higher in RAW264.7 cells after treated with IL-11 compared with control group (Fig. 5A-C). And pre-treated with FD or IL-11RA-siRNA 
transfection ameliorated the protein expressions of IL-11RA and gp130. While Western blotting analysis showed that the expression of IL-11RA was not affected by IL-11, FD or MEK inhibitor in NHLFs (Fig. 5D, E), the protein expression of gp130 was not influenced by IL-11, FD, IL-11RA-siRNA transfection or MEK inhibitor in NHLFs (Fig. 5D, F).

\section{Fluorofenidone Suppressed MEK/ERK Pathway Activation Both in vivo and in vitro}

Two important members of RAS-RAF-MEK-ERK signaling pathway are MEK and ERK. In our study, the expressions of phosphorylated MEK and ERK in the BLM group and in the IL-11 group were remarkably higher compared with those of the control group (Fig. 4A-C; 6A-F). And FD pre-treatment notably decreased these changes (Fig. 4A-C; 6A-F). Meanwhile, neutralizing anti-IL-11 antibodies or MEK inhibitor could suppress the phosphorylated MEK and ERK induced by BLM in lung tissues (Fig. 4A-C). IL-11RAsiRNA transfection or MEK inhibitor treatment attenuated the protein expressions of phosphorylation of MEK and ERK (Fig. 6A-F).

\section{Fluorofenidone Decreases IL-11-induced Levels Fibronectin, Collagen $₫$ and a-SMA Protein and/or mRNA in NHLFs}

The main corresponding makers about activation of fibroblasts and deposition of ECM are fibronectin, collagen $\triangle$ and $a-S M A$. The results showed that the protein and/or mRNA expressions of fibronectin, Collagen $\triangle$ and $a-S M A$ in the IL-11 group were significantly increased compared with the control group, whereas FD pre-treatment, IL-11RA-siRNA transfection or MEK inhibitor remarkly inhibited the increase (Fig. 8A-F).

\section{Discussion}

The etiology and pathogenesis of IPF are still unclear. Currently, only two antifibrotic drugs, pirfenidone and nintedanib are now approved for the treatment of IPF. But IPF is a progressive and fatal interstitial lung disease despite medical treatment. Hence, it is particularly important to further explore the pathogenesis of IPF and develop new antifibrotic drugs. Fluorofenidone is a newly synthesized antifibrotic drug that is developed and patented by the Pharmaceutical School of Central South University ${ }^{10}$. Our previous results showed that FD can attenuate pulmonary inflammation and fibrosis in vivo and in vitro ${ }^{11,12,13}$. However, further research is still needed to explore the mechanism of action of FD. Current studies have found that IL- 11 exerts a more important role than TGF - $\beta 1$ in the process of fibrosis. The levels of IL-11 is significantly increase in the lung and fibroblasts from patients with IPF, associated with the extent of fibrosis, and inhibiting the activation of IL-11/ERK signaling pathway can alleviate pulmonary fibrosis ${ }^{6}$. In this study, we proved that FD could ameliorate pulmonary inflammation and fibrosis through suppressing the activation of IL-11/MEK/ERK signaling pathway in vivo and in vitro.

IL-11 has been considered as a necessary downstream factor for a variety of fibrogenic stimuli, such as TGF- $\beta$, PDGF, Angll and so on ${ }^{17}$. Moreover, IL-11 induces the production of extracellular matrix, 
accelerates the migration of myofibroblasts in stromal cells ${ }^{17}$. Furthermore, IL-11 has a strong proinflammatory effect through activating fibroblasts and myofibroblasts to secrete chemokines and cytokines ${ }^{17}$. The results we present here showed that FD could alleviate BLM-induced inflammation response including inflammation cells infiltration, the production of cytokines and chemokines. And FD inhibited BLM-induced fibrosis reaction, such as the excessive deposition of collagen in the interstitium, the expressions of fibronectin, collagen \and a-SMA. Besides, intraperitoneal injection of neutralizing anti-IL-11 antibodies or MEK inhibitor presented the same results as FD. Galuppo, M. and colleagues show the similar findings that MEK inhibitor ameliorates BLM-induced lung injury and fibrosis ${ }^{18}$. Interestingly, we found that FD decreased the level of IL-11 in the bleomycin mouse model.

The typical IL-11 signal transduction pathway occurs through the molecular interactions between IL-11 and IL-11RA1, and between IL-11RA1 and GP130 that forms a hexameric complex by interacting with a second trimer ${ }^{8}$. The expressions of IL-11 and IL-11RA are highly specific to fibroblasts ${ }^{5}$. IL- 11 promotes the synthesis of fibrogenic protein by driving ERK-dependent autocrine signaling ${ }^{5}$ At the past, there were many studies about the therapeutic methods for inhibiting IL-11 signaling pathway including soluble GP130, modified proteins termed muteins and so on ${ }^{19,20,21,22}$. Intraperitoneal injection of neutralizing anti-IL-11 antibodies (X203) attenuates BLM-induced lung fibrosis, and the pulmonary fibrosis is alleviated in the bleomycin II11 ra $1^{-/-}$mouse model ${ }^{6}$. In our study, we found that BLM induced the expressions of IL-11RA, gp130, phosphorylated MEK and ERK, and FD, neutralizing anti-IL-11 antibodies or MEK inhibitor pretreatment could suppress the levels of phosphorylated MEK and ERK.

To further investigate whether IL-11 mediates the proinflammatory response and the profibrotic effect by activating MEK/ERK signaling pathway, and explore the mechanism of FD, the RAW264.7 cells and NHLFs were treated with IL-11, IL-11 plus FD, IL-11 plus IL-11RA-siRNA, IL-11 plus MEK inhibitor. Our work indicated that FD and IL-11RA-siRNA could ameliorate IL-11-induced expressions of IL-11RA and gp130 in RAW264.7 cells. Meanwhile, we found that FD, IL-11RA-siRNA or MEK Inhibitor effectively inhibited IL-11induced activation of MEK and ERK in RAW264.7 cells and in NHLFs. Furthermore, IL-11 led to the production of inflammatory factors, the activation of fibroblasts and deposition of ECM, and FD, IL-11RAsiRNA or MEK Inhibitor attenuated IL-11-induced production of inflammatory factors of IL-8, IL-18, MCP-1,

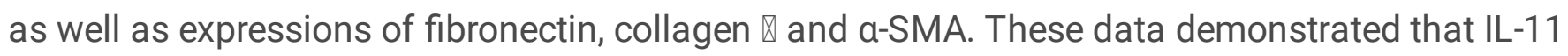
promoted the RAW264.7 cells to secrete inflammatory factors and chemical factor, and activated the MEK/ERK signaling pathway, and induced the activation of fibroblasts. The treatment of FD attenuated IL-11-induced the series of reactions in vitro.

A growing amount of research show that IL-11 is a therapeutically target for IPF. Taken together, our experiments indicate that FD decreases the secretion of IL-11, attenuates lung inflammation and fibrosis through inhibiting the activation of IL-11/MEK/ERK signaling pathway. IL-11 maybe is a molecular target of FD. And we look forward to develop more antifibrotic drugs with fewer side effects, such as the targeted drug for IL-11, which may bring greater benefits for IPF patients. 


\section{Conclusion}

FD attenuates lung inflammation and fibrosis through inhibiting the activation of IL-11/MEK/ERK signaling pathway. However, the pharmacology mechanism and the molecular target of FD are not well understood, further studies should be conducted to identify it.

\section{Declarations}

\section{Acknowledgments}

Guarantors: Cheng Song and Yi Hu take responsibility for the content of the manuscript, including the data and analysis.

\section{Author contributions}

CS conceived and designed the experiments; $\mathrm{YH}$ and SG guided the design of the research. CS, XFL, WJT, XQG, YHM and QZ performed the experiments. CS, XFL, WJT and XQG contributed to reagents/materials/analysis tools; CS analyzed the data and drafted the manuscript. All authors have read and agreed to the final version of this manuscript.

\section{Funding}

This work was supported by the National Natural Science Foundation of China (grant no. 81800064 to C.S.), the Wuhan Municipal Science and Technology Bureau (grant no. 2020020601012322 to C.S.) and the Wuhan Health and Health Commission (grant no. WX20Q11 to C.S.).

\section{Availability of data and materials}

The datasets used and/or analyzed during the current study are available from the corresponding author on reasonable request.

\section{Ethics approval}

The animal experiments were reviewed and approved by Laboratory Animal Center, Huazhong University of Science and Technology.

\section{Consent for publication}

Not applicable.

\section{Competing interests}

The authors declare that they have no competing interests.

\section{References}


1. Raghu, G., et al., An official ATS/ERS/JRS/ALAT statement: idiopathic pulmonary fibrosis: evidencebased guidelines for diagnosis and management. Am J Respir Crit Care Med, 2011. 183(6): p. 788824.

2. King, T.J., A. Pardo and M. Selman, Idiopathic pulmonary fibrosis. Lancet, 2011. 378(9807): p. 194961.

3. Barron, L. and T.A. Wynn, Fibrosis is regulated by Th2 and Th17 responses and by dynamic interactions between fibroblasts and macrophages. Am J Physiol Gastrointest Liver Physiol, 2011. 300(5): p. G723-8.

4. Henderson, N.C., F. Rieder and T.A. Wynn, Fibrosis: from mechanisms to medicines. Nature, 2020. 587(7835): p. 555-566.

5. Schafer, S., et al., IL-11 is a crucial determinant of cardiovascular fibrosis. Nature, 2017. 552(7683): p. 110-115.

6. Ng, B., et al., Interleukin-11 is a therapeutic target in idiopathic pulmonary fibrosis. Sci Transl Med, 2019. 11(511).

7. Lokau, J., et al., Proteolytic Cleavage Governs Interleukin-11 Trans-signaling. Cell Rep, 2016. 14(7): p. 1761-1773.

8. Nguyen, P.M., S.M. Abdirahman and T.L. Putoczki, Emerging roles for Interleukin-11 in disease. Growth Factors, 2019. 37(1-2): p. 1-11.

9. Silver, J.S. and C.A. Hunter, gp130 at the nexus of inflammation, autoimmunity, and cancer. J Leukoc Biol, 2010. 88(6): p. 1145-56.

10. Lou, Q., et al., Design, synthesis and antifibrotic activities of carbohydrate-modified 1-(substituted aryl)-5-trifluoromethyl-2(1H) pyridones. Molecules, 2012. 17(1): p. 884-96.

11. Meng, J., et al., Fluorofenidone attenuates bleomycin-induced pulmonary inflammation and fibrosis in mice via restoring caveolin 1 expression and inhibiting mitogen-activated protein kinase signaling pathway. Shock, 2012. 38(5): p. 567-73.

12. Liu, J., et al., Fluorofenidone attenuates TGF-beta1-induced lung fibroblast activation via restoring the expression of caveolin-1. Shock, 2015. 43(2): p. 201-7.

13. Song, C., et al., Fluorofenidone attenuates pulmonary inflammation and fibrosis via inhibiting the activation of NALP3 inflammasome and IL-1beta/IL-1R1/MyD88/NF-kappaB pathway. J Cell Mol Med, 2016. 20(11): p. 2064-2077.

14. Szapiel, S.V., et al., Bleomycin-induced interstitial pulmonary disease in the nude, athymic mouse. Am Rev Respir Dis, 1979. 120(4): p. 893-9.

15. Ashcroft, T., J.M. Simpson and V. Timbrell, Simple method of estimating severity of pulmonary fibrosis on a numerical scale. J Clin Pathol, 1988. 41(4): p. 467-70.

16. Lv, X., et al., Protective Effect of Fluorofenidone Against Acute Lung Injury Through Suppressing the MAPK/NF-kappaB Pathway. Front Pharmacol, 2021. 12: p. 772031. 
17. Cook, S.A. and S. Schafer, Hiding in Plain Sight: Interleukin-11 Emerges as a Master Regulator of Fibrosis, Tissue Integrity, and Stromal Inflammation. Annu Rev Med, 2020. 71: p. 263-276.

18. Galuppo, M., et al., MEK inhibition suppresses the development of lung fibrosis in the bleomycin model. Naunyn Schmiedebergs Arch Pharmacol, 2011. 384(1): p. 21-37.

19. Putoczki, T.L., et al., Interleukin-11 is the dominant IL- 6 family cytokine during gastrointestinal tumorigenesis and can be targeted therapeutically. Cancer Cell, 2013. 24(2): p. 257-71.

20. Sano, E., et al., Production and characterization of genetically modified human IL-11 variants. Biochim Biophys Acta Gen Subj, 2017. 1861(2): p. 205-217.

21. Deguchi, Y., et al., Generation of and characterization of anti-IL-11 antibodies using newly established II11-deficient mice. Biochem Biophys Res Commun, 2018. 505(2): p. 453-459.

22. Lamertz, L., et al., Soluble gp130 prevents interleukin-6 and interleukin-11 cluster signaling but not intracellular autocrine responses. Sci Signal, 2018. 11(550).

\section{Figures}


A

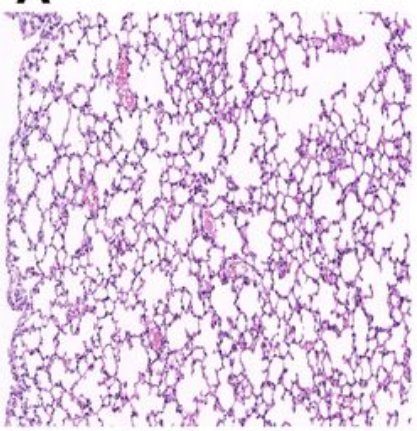

D

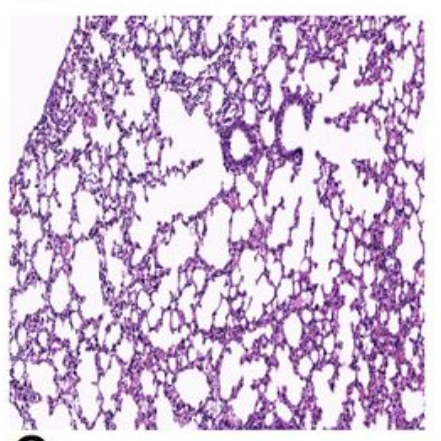

G

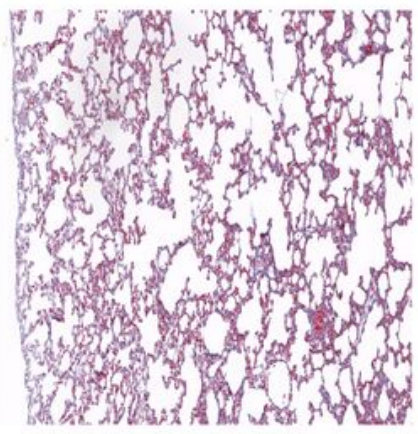

J

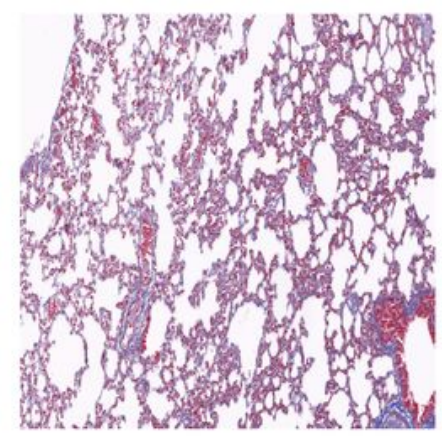



E

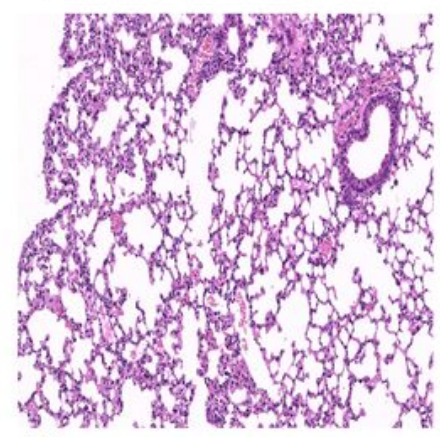

H

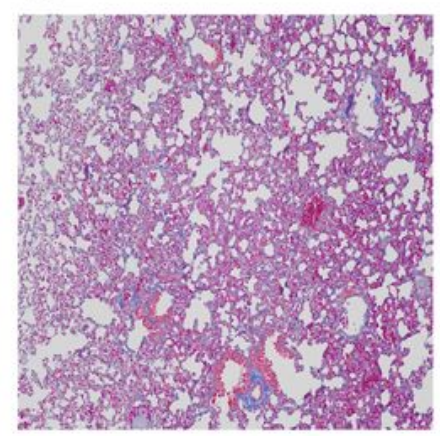

K

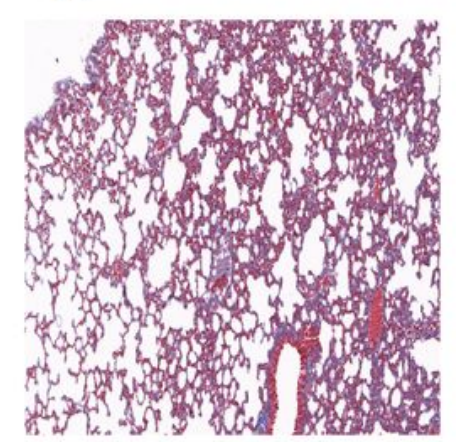

C

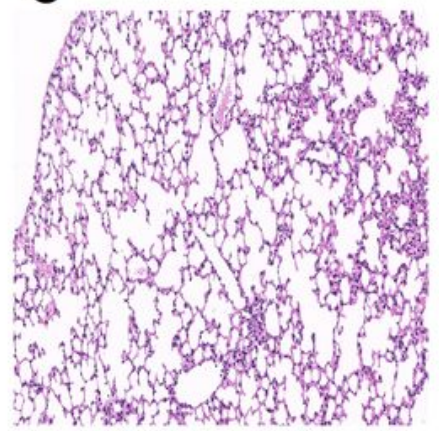

F

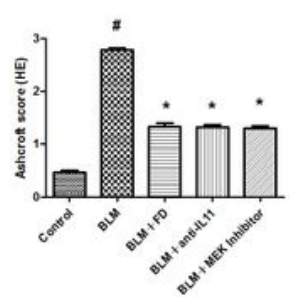

I

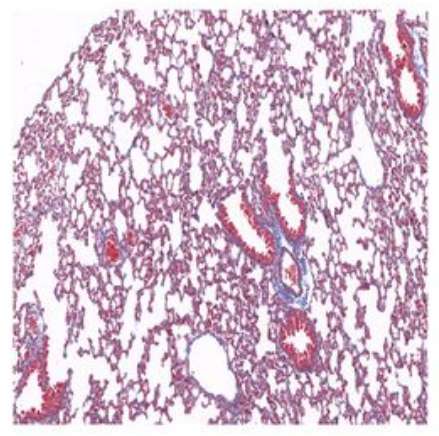

L

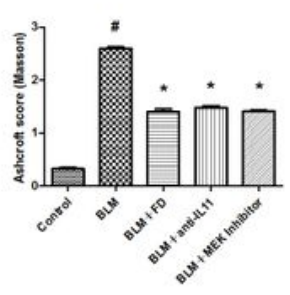

\section{Figure 1}

Fluorofenidone attenuated pulmonary inflammation and fibrosis in the bleomycin mouse model

Representative micrographs (H囚E staining, 200x) of lungs from control mice (A), BLM mice (B), BLM+FD mice (C), BLM+anti-IL-11 mice (D) and BLM+MEK inhibitor mice (E) were showed. Alveolitis scores (F). Representative micrographs (Masson's Trichrome staining, 200x) of lungs from control mice (G), BLM 
mice $(\mathrm{H}), \mathrm{BLM}+\mathrm{FD}$ mice $(\mathrm{I}), \mathrm{BLM}+\mathrm{anti}-\mathrm{IL}-11$ mice $(\mathrm{J})$ and BLM+MEK inhibitor mice $(\mathrm{K})$ were showed. Ashcroft scores $(L)$. Results are expressed as Mean $\pm S D, n=8$ mice in control, $B L M$ or $B L M+F D$ groups,

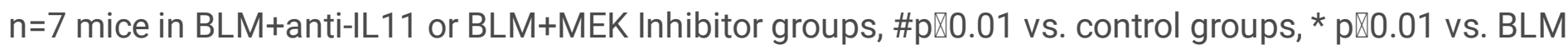
groups.

A

A



BLM

BLM+FD

BLM+anti-IL11

BLM+MEK
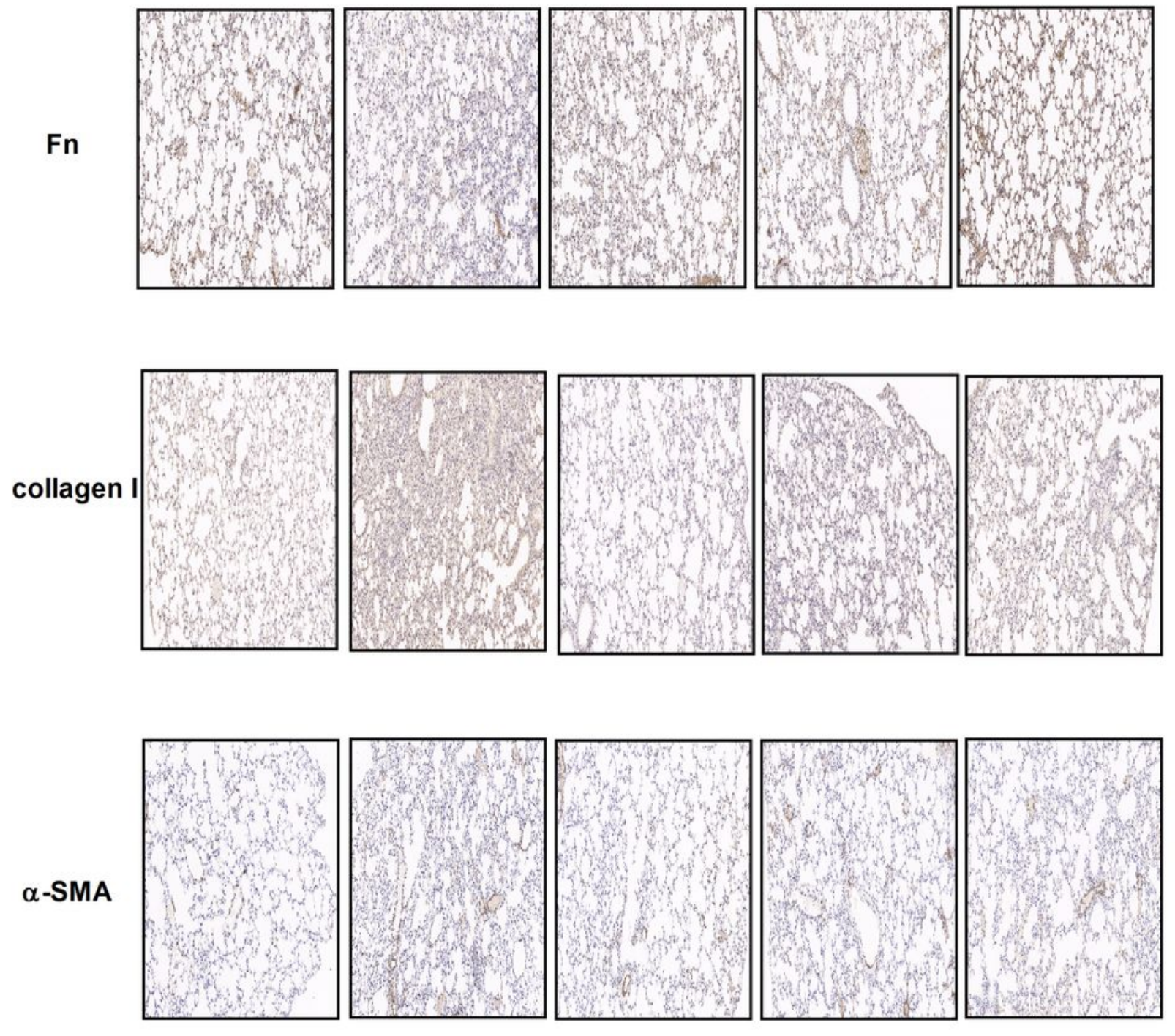

B

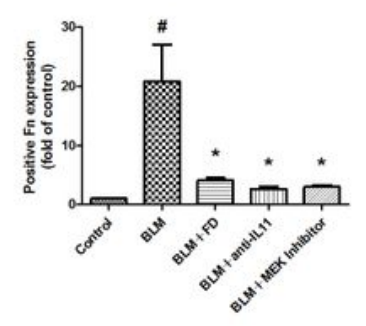

C

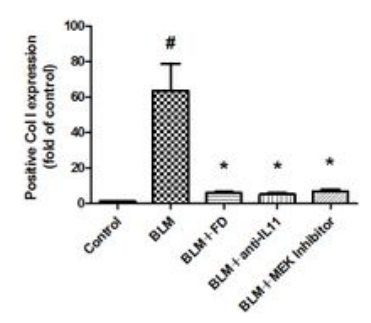

D

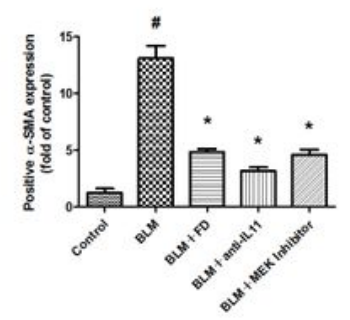

Figure 2 
Fluorofenidone ameliorated the expressions of fibronectin, collagen $₫$ and a-SMA in the bleomycin mouse model

Representative images (A) and quantitative analysis of immunehistochemistry (200x) for Fibronectin (B), Collagen $\otimes(C)$ and $a-S M A(D)$ in lung tissues. Results are expressed as Mean $\pm S D, n=8$ mice in control, BLM or BLM+FD groups, $\mathrm{n}=7$ mice in BLM+anti-IL11 or BLM+MEK Inhibitor groups, ${ }^{\#} \mathbb{p} \otimes 0.01$ vs. control groups, * p $₫ 0.01$ vs. BLM groups.

A



C

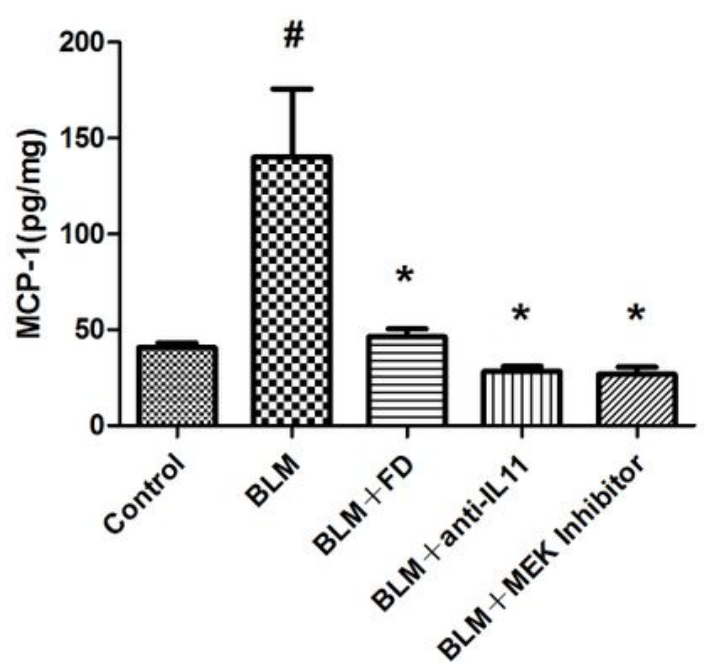

B

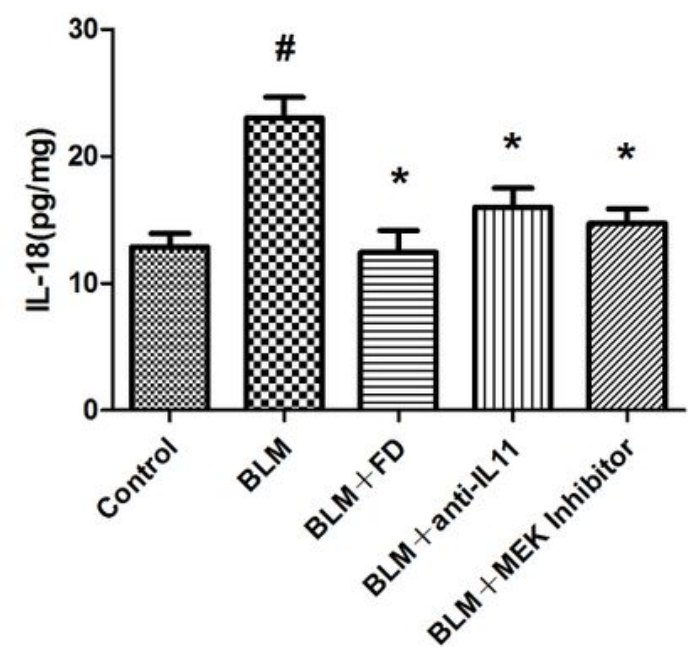

D

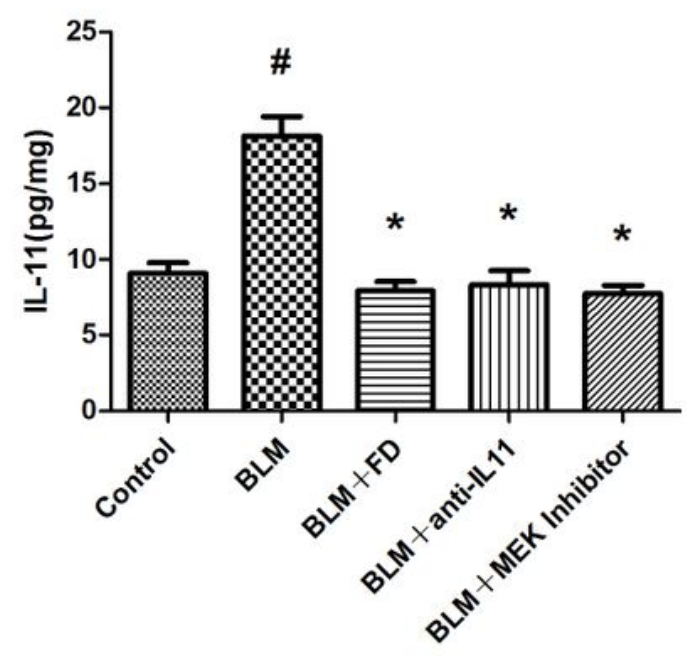


Figure 3

Fluorofenidone decreased the levels of inflammatory factors in blood of mice

(A) IL-8, (B) IL-18, (C) MCP-1, (D) IL-11 concentrations in blood of mice. Results are expressed as Mean \pm $S D, n=6$ mice per group, ${ }^{*} \mathrm{p} \otimes 0.01$ vs. control groups, * $\mathrm{p} \otimes 0.01$ vs. BLM groups.

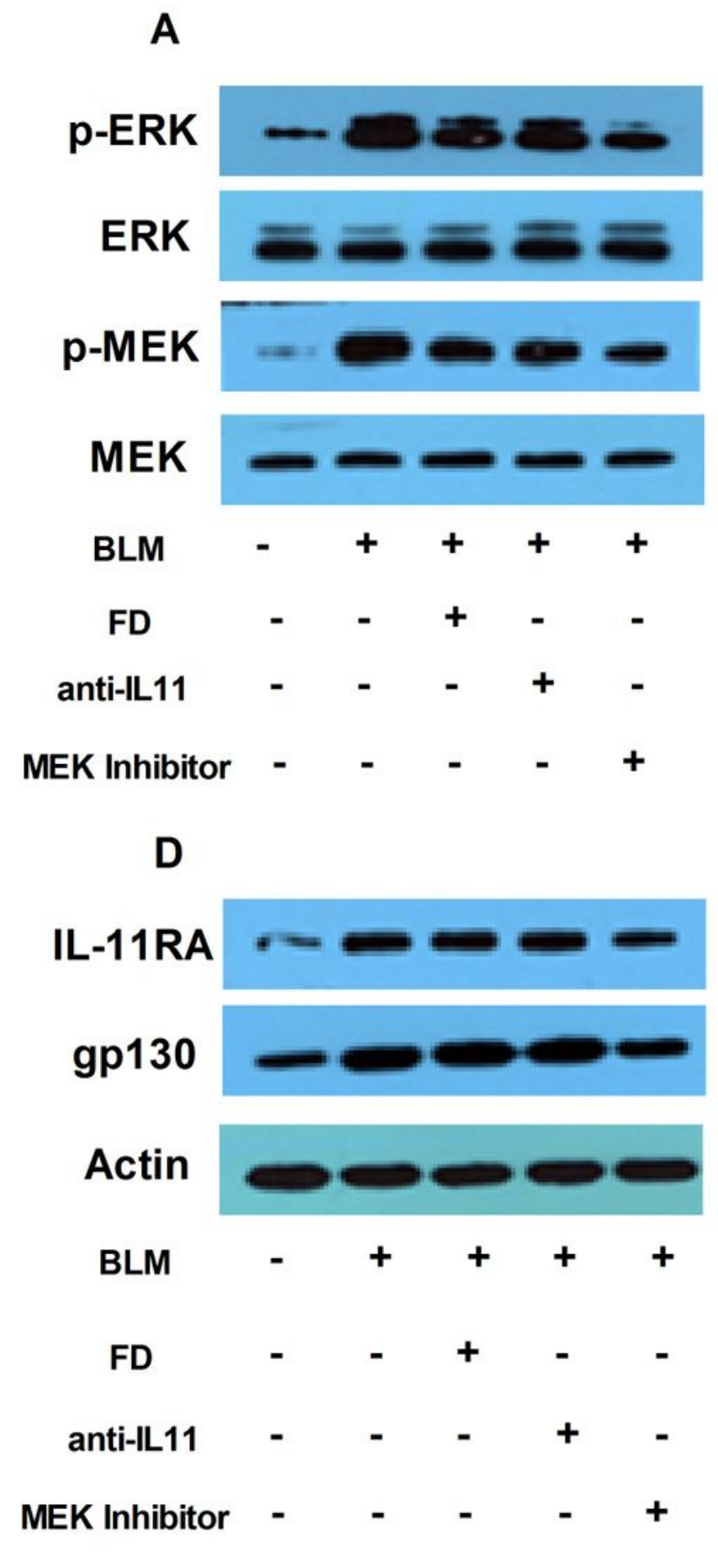

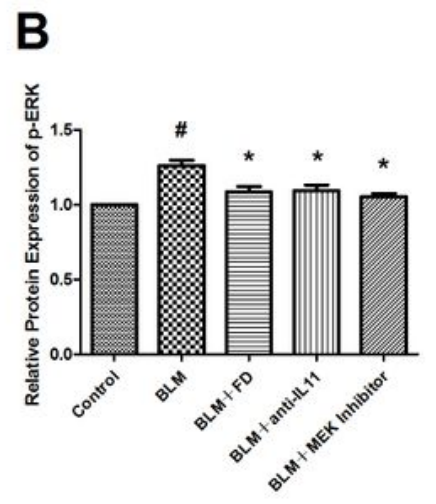
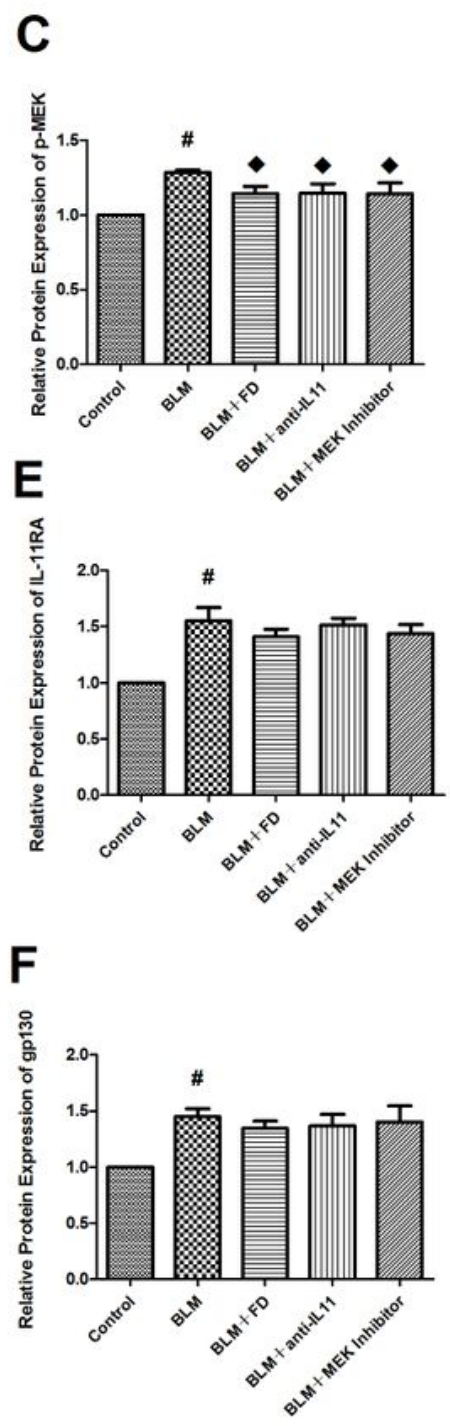
Fluorofenidone attenuates BLM-induced activation of MEK and ERK, but cannot downregulate BLMinduced expressions of IL-11RA and gp130

The expressions of IL-11RA, gp130, MEK, p-MEK, ERK, p-ERK were measured by Western blotting. (A) representative Western blot of p-ERK, ERK, p-MEK and MEK, (B) quantitative analysis of $p$-ERK, (C) quantitative analysis of p-MEK, (D) representative Western blot of IL-11RA, gp130, (E) quantitative analysis of IL-11RA, (F) quantitative analysis of gp130. Results are expressed as Mean $\pm S D, n=5$ mice

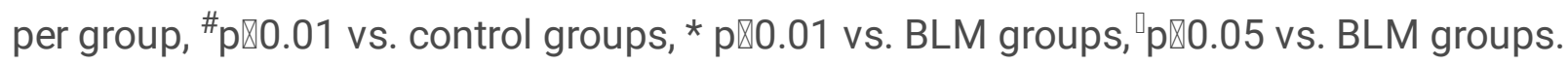


A
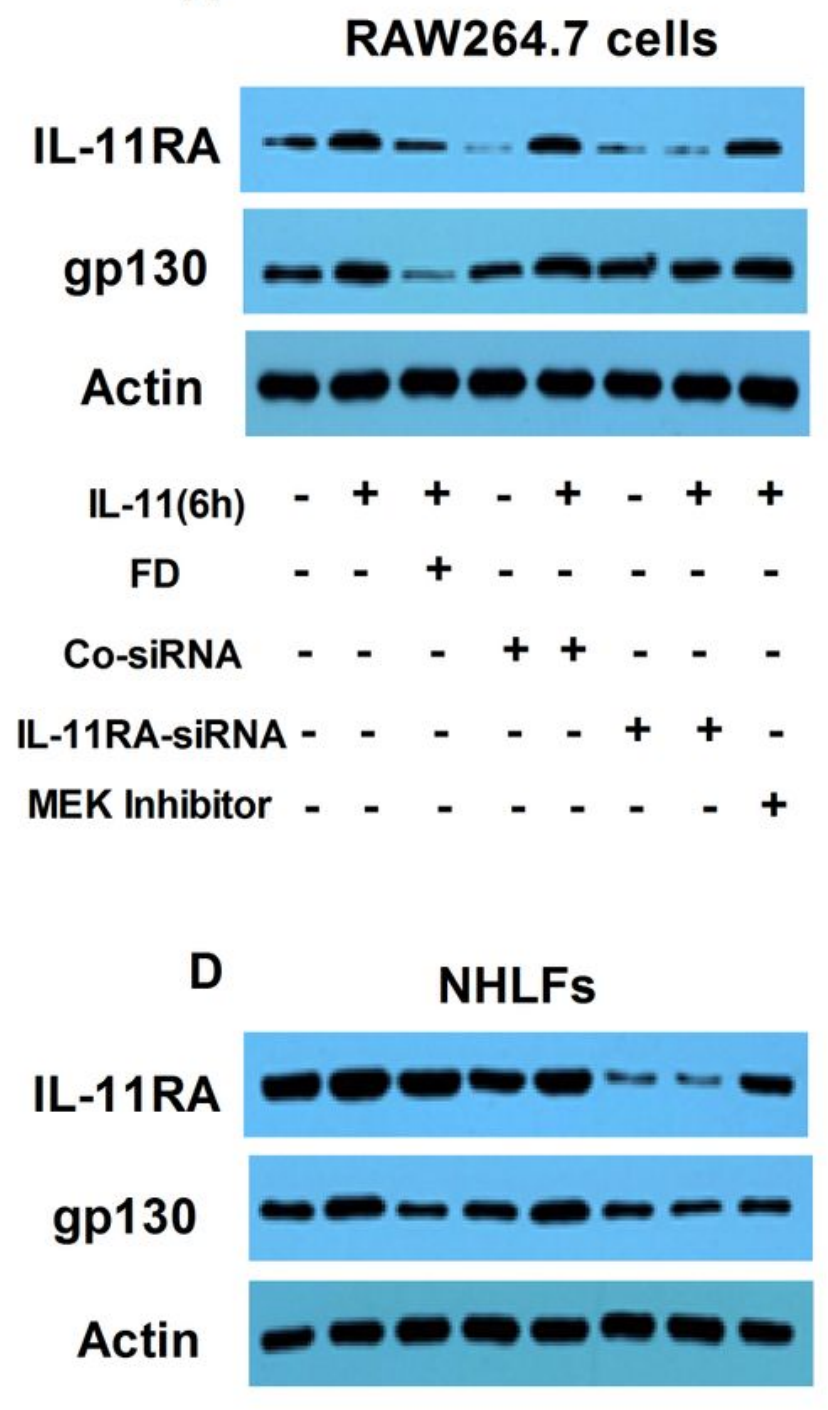

$\mathrm{IL}-11(48 \mathrm{~h}) \quad-\quad+\quad+\quad+\quad+\quad+$

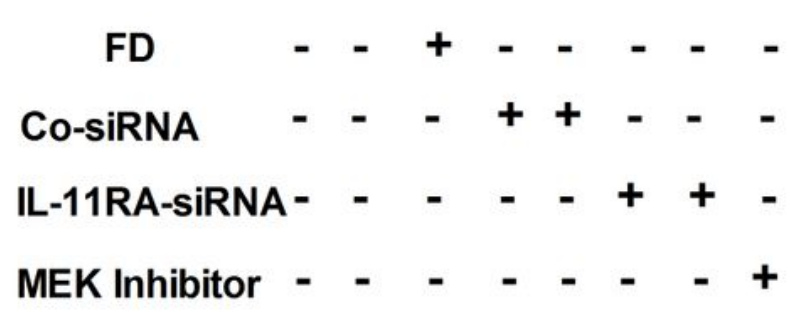

B

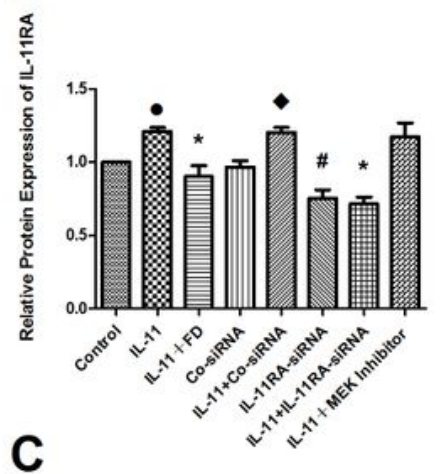

C



$\mathbf{E}$

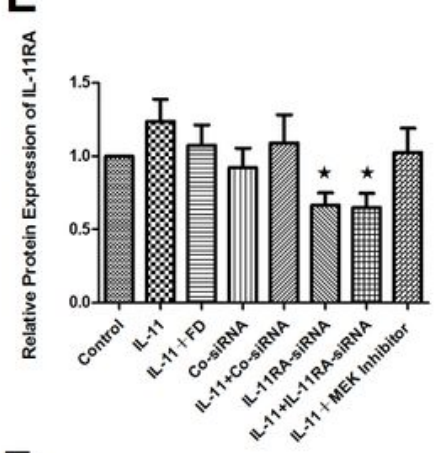

$\mathbf{F}$

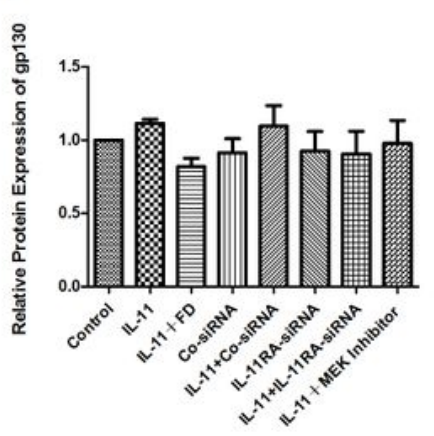

Figure 5

Fluorofenidone decreases IL-11-induced levels of IL-11RA and gp130 in RAW264.7 cells, but cannot in NHLFs

(A), representative Western blot of IL-11RA and gp130 in RAW264.7 cells, (B), quantitative analysis of IL11RA in RAW264.7 cells, (C), quantitative analysis of gp130 in RAW264.7 cells, (D), representative 
Western blot of IL-11RA and gp130 in NHLFs, (E), quantitative analysis of IL-11RA in NHLFs, (F),

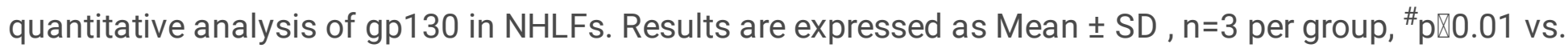
control groups, ${ }^{\bullet} \mathrm{p} \varangle 0.05$ vs. control groups, ${ }^{\square} \mathrm{p} \rrbracket 0.01$ vs. Co-siRNA groups, ${ }^{*} \mathrm{p} \rrbracket 0.01$ vs. IL-11 groups, ${ }^{\Delta} \mathrm{p} \rrbracket$ 0.05 vs. IL-11 groups, $\nabla \mathrm{p} \otimes 0.05$ vs. IL-11+IL-11RA-siRNA groups, ${ }^{\natural} \rrbracket 0.05$ vs. IL-11 groups, FD groups and IL-11+Co-siRNA groups.

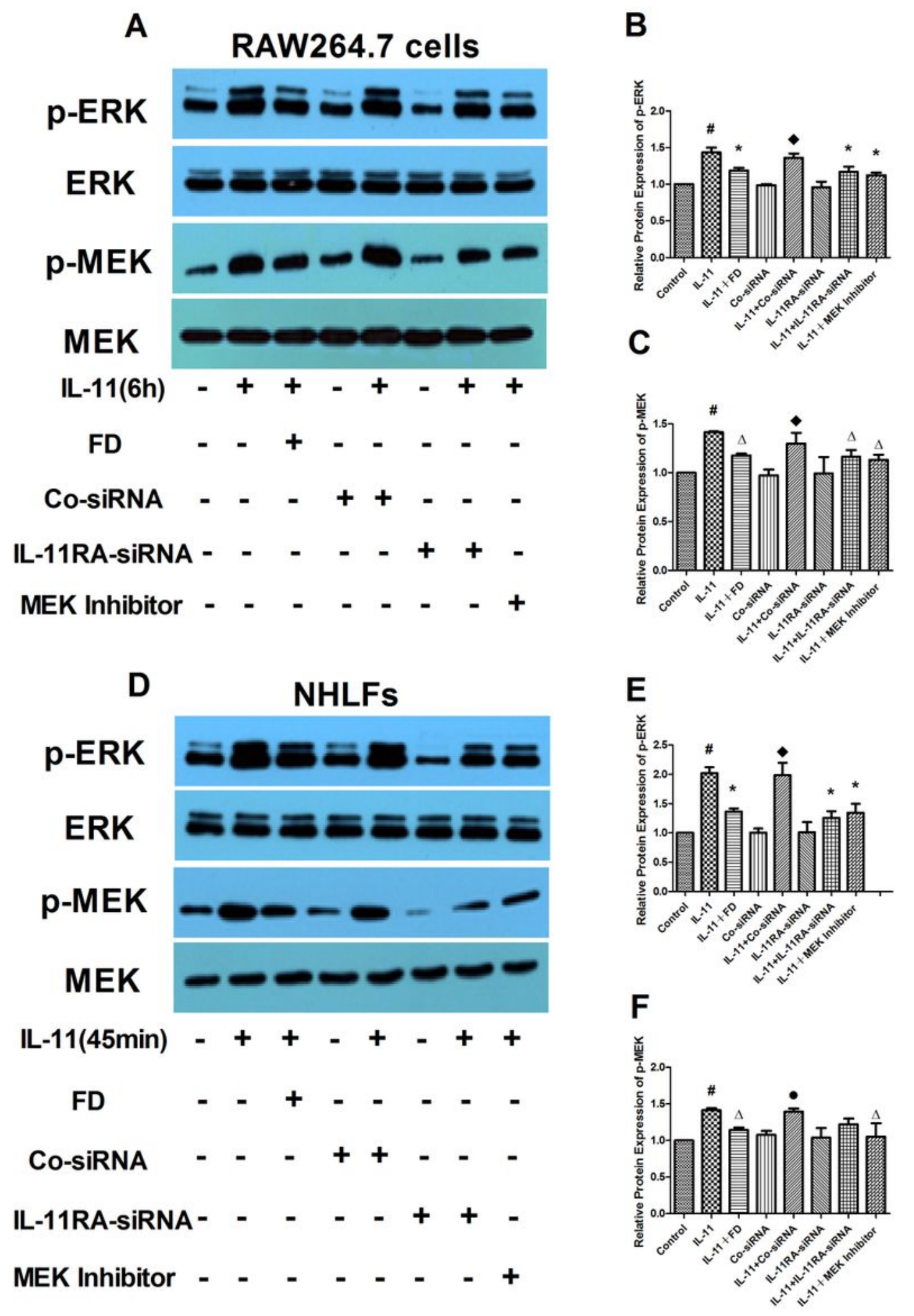

Figure 6 
(A), representative Western blot of p-ERK, ERK, p-MEK and MEK in RAW264.7 cells, (B), quantitative analysis of p-ERK in RAW264.7 cells, (C), quantitative analysis of p-MEK in RAW264.7 cells, (D), representative Western blot of $p$-ERK, ERK, p-MEK and MEK in NHLFs, (E), quantitative analysis of p-ERK in NHLFs, $(F)$, quantitative analysis of $p-M E K$ in NHLFs. Results are expressed as Mean $\pm S D, n=3$ per

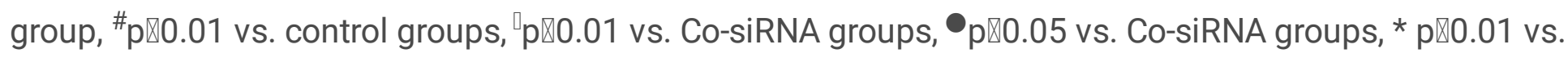


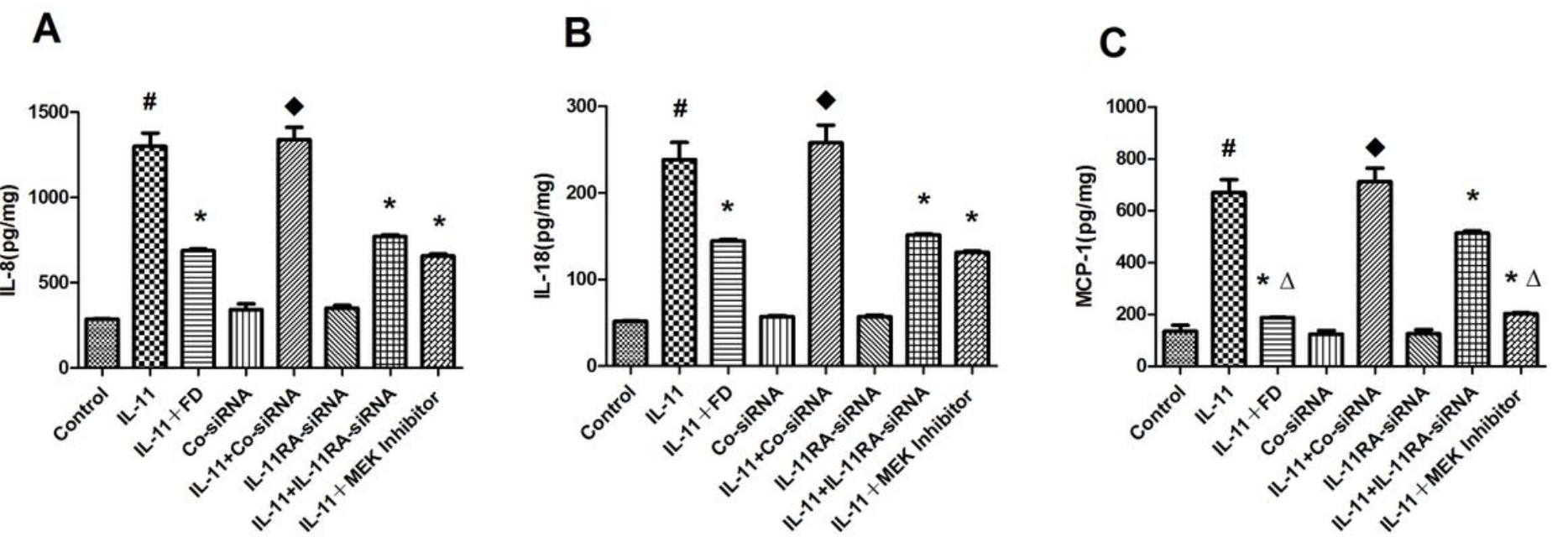

Figure 7

Fluorofenidone inhibits IL-11-induced IL-8, IL-18 and MCP-1 increase in the supernatant fluid of RAW264.7 cells

(A) IL-8, (B) IL-18, (C) MCP-1 concentrations in the supernatant fluid of RAW264.7 cells after IL-11

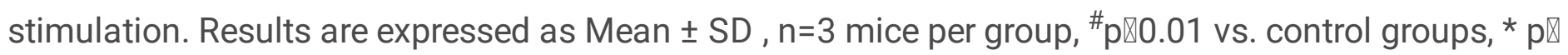
0.01 vs. IL-11 groups, ${ }^{\Delta}$ p $₫ 0.01$ vs. IL-11+IL-11RA-siRNA groups. 

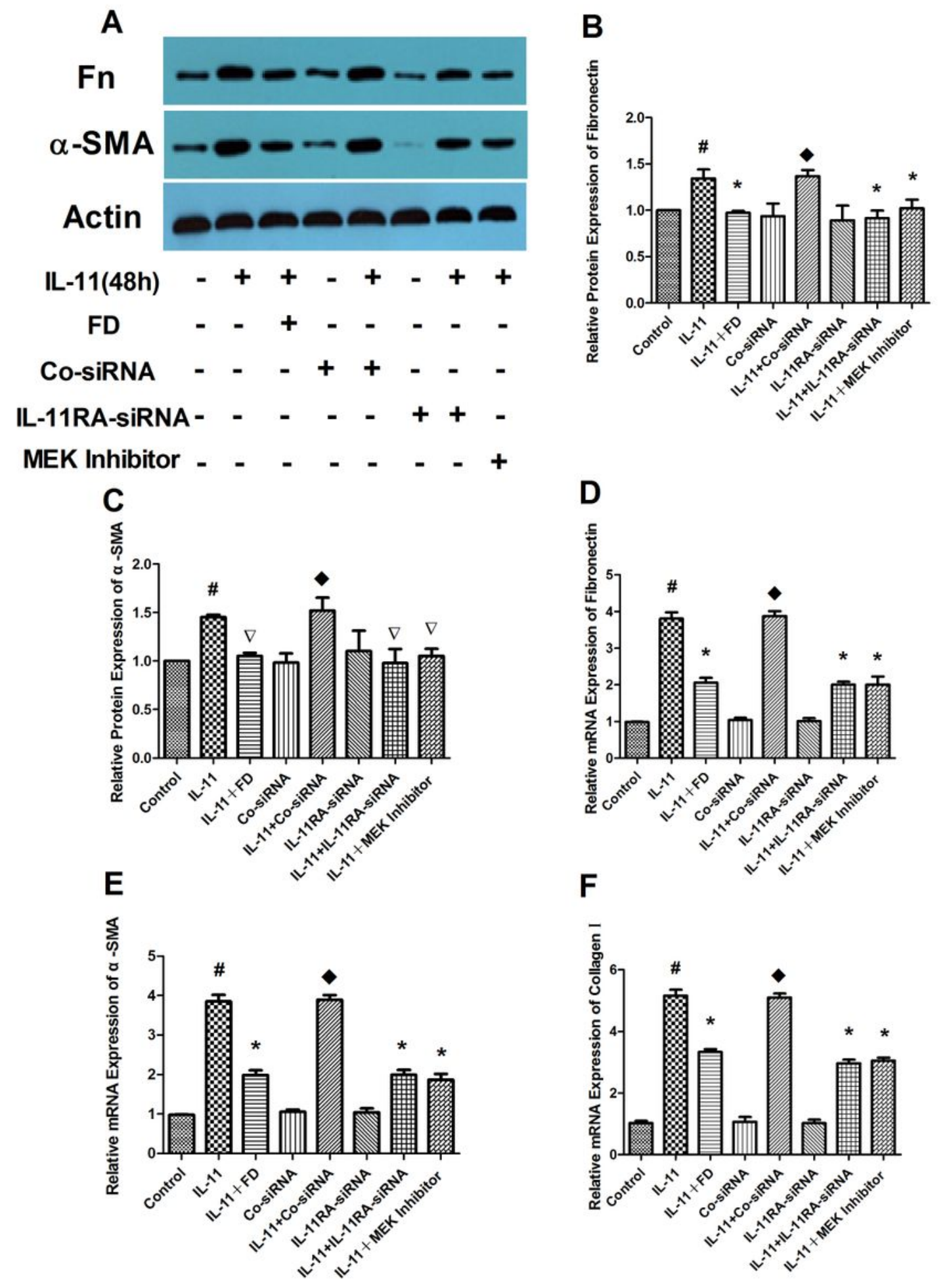

Figure 8

Fluorofenidone decreases IL-11-induced levels fibronectin, collagen $₫$ and a-SMA protein and/or mRNA in NHLFs

(A), representative Western blot of fibronectin and a-SMA, (B), quantitative analysis of fibronectin, (C),

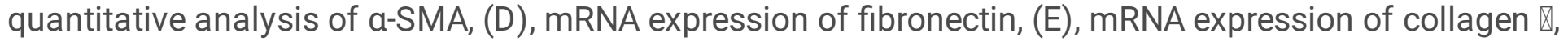




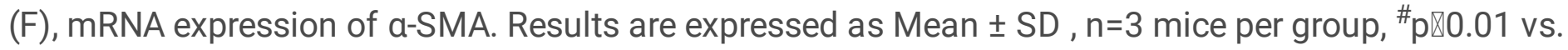

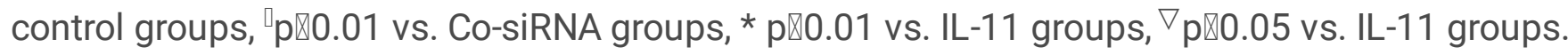

